\title{
Identification of potential crucial genes associated with vasculogenic mimicry in human osteosarcoma based on gene expression profile
}

\author{
N. YAO ${ }^{1,2, *}$, K. REN ${ }^{3, *}$, X. J. GU ${ }^{4}$, S. J. WU ${ }^{5}$, X. SHI ${ }^{5}$, Q. CHANG ${ }^{3}$, Y.G. LI ${ }^{3}$, Z. X. GAO ${ }^{3}$, Q. M. JIN ${ }^{1,2}$, J. ZHANG ${ }^{1,2}$, C. WANG ${ }^{3, *}$, J. ZHOU ${ }^{1,2,6 *}$ \\ ${ }^{1}$ Affiliated Hospital of Integrated Traditional Chinese and Western Medicine, Nanjing University of Chinese Medicine, Nanjing 210028, Jiangsu, \\ China; ${ }^{2}$ Laboratory of Translational Medicine, Jiangsu Province Academy of Traditional Chinese Medicine, Nanjing 210028, Jiangsu, China; \\ ${ }^{3}$ Department of Orthopedics, Zhongda Hospital, Southeast University, Nanjing, 210009, Jiangsu, China; ${ }^{4}$ Institute of Biotechnology, School of \\ Environmental and Chemical Engineering, Dalian Jiaotong University, Dalian 116028, Liaoning, China; ${ }^{5}$ Department of Orthopedics, Jinling \\ Hospital, Nanjing University, School of Medicine, Nanjing 210002, Jiangsu Province, China; ${ }^{6}$ School of Basic Medicine and Clinical Pharmacy, \\ China Pharmaceutical University, Nanjing 211198, Jiangsu Province, China
}

*Correspondence: chenwang_southeast@126.com; happyjingzhou@126.com

${ }^{\#}$ Contributed equally to the work.

Received April 14, 2019 / Accepted July 9, 2019

\begin{abstract}
We previously reported the presence of vasculogenic mimicry (VM) in human osteosarcoma. However, the mechanistic basis of osteosarcoma VM remains unclear. Three hundred eighty-one upregulated differentially expressed genes (DEGs) and 526 downregulated DEGs between human osteosarcoma cell line 143B and HOS cell exposed to Matrigel were screened out by microarray. GO categories such as "cell adhesion", "angiogenesis" were enriched in 143B group. PATHWAY analysis showed enriched TGF-beta, Wnt and VEGF signaling pathway in 143B group. The hub gene ITGA2 in signal-network of DEGs exhibited pro-VM and pro-metastasis effect. Our study provides fundamental data for further studies regarding molecules involved in osteosarcoma VM.
\end{abstract}

Key words: osteosarcoma, microarray analysis, neoplasm metastasis

In 1999, Maniotis et al. [1] first reported a novel phenomenon in tumor angiogenesis: vasculogenic mimicry (VM). It describes functional plasticity of highly aggressive and invasive tumor cells in dedifferentiating to a vascular phenotype and forming de novo tube-like networks, thus distributing red blood cells and nutrition to malignant tumor growth independent of conventional vasculogenesis or angiogenesis. It also provides a unique structure in which the inner surface of the networks exposes tumor cells direct to the blood stream, thus greatly promoting tumor metastasis to distant sites [2]. These tubular networks could also be observed in vitro with highly invasive tumor cells in three-dimensional culture conditions, which comprised of extracellular matrix (ECM) and resemblances with tube-like structures observed in classic endothelial cell-based in vitro angiogenesis assay [3].

Since its first report in melanoma, VM has been involved in a variety of malignant human tumors including osteosarcoma [4]. VM is associated with poor clinical outcome, reduced patient survival and increased risk of metastasis [2]. These findings were consistent with our previous report that in patients with osteosarcoma, VM is associated with decreased overall survival rates and the development of distant metastasis, thus serving as an unfavorable prognostic factor for osteosarcoma patients [4]. Similarly, there is a correlation between the histological detection of VM in in vivo osteosarcoma xenograft model and subsequent death from metastasis, in accordance with the in vitro observations showing that VM channels are formed by highly invasive osteosarcoma cells $[5,6]$.

Microarray studies comparing VM-positive aggressive tumor cells and non-VM tumor cells revealed multiple signaling molecules and cascades involved in VM. For example, VE-cadherin and EPHA2 are two of the first molecules identified to play a key role in mediating VM [7]. Other molecules including Galectin-3, cAMP, COX-2 and MIG-7 have been reported to be engaged in VM [8]. Signaling pathway like HIF-1 $\alpha$, Notch/Nodal and Wnt/ $\beta$-catenin were shown to contribute to VM formation [8]. Targeting VM with specific compounds may become a promising therapeutic approach against tumor progression [9]. However, the mechanism of osteosarcoma VM remains unclear since its 
first report in 2004. It is therefore of extreme importance and urgent demand to explore the molecules underlying osteosarcoma VM to define potential genetic targets for therapeutic development against osteosarcoma.

It is well known that microarray-based gene expression profiling studies in cancer provide a high-throughput, efficient approach to gain a wealth of information with potential diagnostic and prognostic uses and to investigate the mechanisms that underscore tumorigenic phenotype. Because VM formation requires tumor cells cultured in an ECM-rich microenvironment, we herein compared gene expression profiles between commonly used human metastatic 143B osteosarcoma cell line and non-metastatic HOS osteosarcoma cell line in three dimensional Matrigel matrices for two reasons: firstly, 143B cells could form VM channels on Matrigel, an ECM extract culture medium used for in vitro tube formation assay [5], whereas HOS cells could not (Figure 1). Secondly, as many human osteosarcoma cell lines are derived from different genetic origins, we therefore applied commonly used human metastatic osteosarcoma cell line $143 \mathrm{~B}$ and its parental non-metastatic lines HOS to avoid interference from different genetic background.

In the present study, we performed gene expression profiling to identify differentially expressed genes (DEGs) between 143B cells and HOS cells cultured on Matrigel to fully understand VM phenotypes at the molecular level. Then, we analyzed their functions by GSEA GO analysis and GSEA pathway analysis. Signal network (Signal-net) was conducted to show hub genes that might play critical roles in osteosarcoma VM formation. Finally, we demonstrated a top-ranked hub gene ITGA2 had a VM-promoting role in osteosarcoma. This study aimed to shed light on the molecular underpinnings of osteosarcoma VM, and clarify novel therapeutic targets for the development of innovative treatment strategies for osteosarcoma.

\section{Materials and methods}

Cell lines and culture. Human osteosarcoma cells (HOS and 143B) were purchased from the American Type Culture Collection (ATCC, Manassas, VA, USA) and cultivated, according to the recommendation of the supplier, in RPMI 1640 (Invitrogen, Carlsbad, CA, U.S.) containing 10\% FBS (Gibco Life Technologies, Grand Island, NY, U.S.) and DMEM (Invitrogen) containing 10\% FBS, respectively. Streptomycin $(100 \mathrm{mg} / \mathrm{ml})$ and penicillin $(100 \mathrm{U} / \mathrm{ml})$ were added to each medium. All cells were cultured in T75 flasks (Nunc, U.S.) in a humidified incubator at $37^{\circ} \mathrm{C}$ with $5 \%$ $\mathrm{CO}_{2}$, and culture medium exchange was performed every 24 hours.

VM assay. A 6-well plate was evenly coated with $1 \mathrm{ml} /$ well cold Matrigel (BD Biosciences, San Jose, CA, USA, thawed at $4{ }^{\circ} \mathrm{C}$ ) and allowed to solidify at $37^{\circ} \mathrm{C}$ for $30 \mathrm{~min}$. Then cell suspension $\left(6 \times 10^{5} / \mathrm{ml}\right)$ was added $(1 \mathrm{ml} /$ well $)$ onto the surface of the plate and incubated at $37^{\circ} \mathrm{C}$ for $3,8,12$ and
24 h, respectively. Cells were photographed under a light microscope (Primo Vert, Zeiss, Germany).

RNA preparation. Twenty-four hours after osteosarcoma cell lines were seeded on Matrigel, total RNA was extracted using Trizol reagent (Invitrogen, Carlsbad, CA, U.S.) according to the manufacturer's instructions. RNA integrity was assessed by electrophoresis on a denaturing agarose gel. RNA concentration and purity were determined by a NanoDrop 2000 spectrophotometer using $\mathrm{OD}_{260}$ andOD ${ }_{260} /$ $\mathrm{OD}_{280}$ ratio, respectively. The extracted RNA samples were stored at $-80^{\circ} \mathrm{C}$ for further experiments.

Microarray assay. Global gene expression profiling analysis was performed using Affymetrix GeneChip "Human Transcriptome 2.0 Array (HTA2.0, Affymetrix, Santa Clara, CA, USA). Briefly, 250 ng total RNA was reverse transcribed to biotinylated cDNA using GeneChip ${ }^{\oplus}$ WT PLUS Reagent Kit according to the standard Affymetrix protocol. Following labeling, cDNA was hybridized for $16 \mathrm{~h}$ at $45^{\circ} \mathrm{C}$ on GeneChip Human Transcriptome Array 2.0 in GeneChip Hybridization Oven 645 (Affymetrix). GeneChips were washed and stained in the Affymetrix Fluidics Station 450, and then scanned using the Hewlett-Packard GeneArray Scanner 3000 7G. Data was submitted to the Gene Expression Omnibus (GEO) repository (https://www.ncbi.nlm. nih.gov/geo/) with accession number GSE119975 (GEO: GSE119975).

Data preprocessing and DEG identification. The data were analyzed with Robust Multichip Analysis (RMA) algorithm using Affymetrix default analysis settings and global scaling as normalization method. The DEGs between HOS and 143B cells were screened out using the cut-off criteria of fold change (FC) $\geq 3.0$ and $p$-value $<0.05$.

Clustering analysis of DEGs. Hierarchical clustering of DEGs based on the Euclidean distance was built using the $\mathrm{R}$ heatmap package (cran.r-project.org/web/packages/ pheatmap/index.html) and the heat map results were visualized using Heatmap software (version1.0, https://www-hivlanl-gov.vpn.seu.edu.cn/content/sequence/HEATMAP/ heatmap.html).

GSEA analysis. GSEA is a computational approach to identify classes of genes that are over-represented in a large set of genes between two biological states, such as disease phenotypes. GSEA software version 2.2.3 and gene sets obtained from Molecular Signatures Database v5.1 (MsigDB) (http://software.broadinstitute.org/gsea/index.jsp) or from published gene signatures were used for analysis. GSEA starts with a list of genes ranked by using a signal-to-noise metric and according to their differential expression levels across HOS and 143B group. Then enrichment score was calculated based on a weighted Kolmogorov-Smirnov-like statistic. Statistical significance was assessed by comparing the enrichment score to enrichment results generated from 1,000 random permutations of the gene set to obtain $p$-values (nominal p-value). The significant threshold was set at $\mathrm{p}$-value $<0.01$ in this study. 
Signal-net analysis. Signal-net analysis of DEGs was built based on KEGG database to illustrate gene regulatory network. Networks were presented as graphs, where nodes are mainly genes and edges represent relation types between the nodes, such as activation or inhibition. Degree centrality is an index that weighs the importance of a certain gene, including indegree and outdegree. The degree of a node is defined as the number of links between this node itself and all other nodes. Gene with higher degrees implies a greater ability to modulate genes those it correlates with.

qPCR. A total of $1 \mu \mathrm{g}$ RNA used as template was reverse transcribed to first-strand cDNA using a HiScript ${ }^{\circledR}$ II QRT SuperMix for qPCR (Vazyme Biotech Co., Ltd., Nanjing, China). Quantitative PCR was performed in total reaction volume of $20 \mu \mathrm{l}$ using ChamQ ${ }^{\mathrm{mi}}$ Universal SYBR ${ }^{\star}$ qPCR Master Mix (Vazyme). The primers were synthesized by Generay Corporation (Shanghai, China). The sequence of primers was shown in Table S1. The qPCR assays were performed using the QuantStudio Dx instrument (Life Technologies, Foster City, CA). GAPDH was chosen as the endogenous standard. Expression level of individual gene was normalized to GAPDH according to the $2^{-\triangle \Delta C T}$ method.

Western blot. Cells were washed twice with PBS and lysed in lysis buffer at $4^{\circ} \mathrm{C}$. Equal amounts of protein $(30 \mu \mathrm{g})$ were separated by SDS-PAGE and transferred to PVDF membranes. The membrane was incubated with anti-ITGA2 antibody (R\&D systems) and detected by using a DyLight secondary antibody (CST, MA, USA). ITGA2 protein expression was normalized against $\beta$-Actin.

siRNA transfection. The small interfering RNAs (siRNA) targeting ITGA2 were purchased from Thermo Fisher Scientific (Carlsbad, CA). The sequences of siRNA were as follows: siITGA2\#1: 5'-GACCAUUGUCCAGAAGACAUCUCAU-3', siITGA2\#2: 5'-GCCUGCAGAAGAAUAUGGUAGUAAA-3', and a none-related control siRNA (Invitrogen\#12935300) was used as a negative control. Transfections were performed in 143B cells using the Lipofectamine RNAiMAX Reagent (Invitrogen) according to the manufacturer's instructions. The knockdown efficiency was verified $24 \mathrm{~h}$ after transfection.

Lentivirus shRNA knockdown of ITGA2. The ITGA2 shRNA (sequences: 5'-ATGGCAATATCACGGTTATTC-3') or one off-target shRNA (sequences: 5'-TTCTCCGAACGTGTCACGT-3') in the lentiviral expression vector pLenRGPH was purchased from Nanjing KeyGen Biotech Co., Ltd. (Nanjing, China). Then the recombinant lentivirus was produced by co-transfecting 293FT cells with packaging helper plasmids and pLenR-GPH-ITGA2 shRNA or pLenRGPH-NC shRNA vectors. 143B cells transduced with lentivirus ITGA2 shRNA vector or lentivirus shRNA control vector are designated as ITGA2 KD or NC cells. The knockdown of ITGA2 was evaluated by qPCR and Western blot.

In vivo tumor xenograft model. The Institutional Animal Care and Use Committee (IACUC) of the Jiangsu Province
Academy of Traditional Chinese Medicine approved the animal study that was conducted in accordance with the National Institutes of Health Guide for the Care and Use of Laboratory animals. Six-week-old male BALB/C nude mice, purchased from Shanghai lingchang Bio-Tech Co., Ltd. (Certificate no. SCXK (HU) 2017-0005), were intratibially injected with $5 \times 10^{5} \mathrm{NC}$ or ITGA2 KD $143 \mathrm{~B}$ cells in $10 \mu \mathrm{l}$ of PBS. Each group contained eight mice. Tumor volume (V) was measured every 3 days by gauging the length (L) and width $(\mathrm{W})$ of the cell-injected and the healthy control right leg with calipers $\left(\mathrm{V}=\mathrm{V}_{\text {left leg }}-\mathrm{V}_{\text {right leg }}\right.$ leg volume $\left[\mathrm{mm}^{3}\right]=$ $\left.0.5 \times \mathrm{L} \times \mathrm{W}^{2}\right)$. Mice were sacrificed 30 days after injection and the primary tumors and lungs were harvested. Lung metastasis was quantified by counting the number of tumor nodule on lung surface with stereomicroscope (Stemi 2000-C, Zeiss, Germany). Organs were then fixed in formaldehyde for further examination.

Histology analysis. Tumor and lung samples were embedded in paraffin, sectioned $(4 \mu \mathrm{m})$, and stained with Hematoxylin and eosin (H\&E) for general histopathology.

Immunohistochemical analysis and quantification. Paraffin sections were deparaffinized by xylene and rehydrated in decreasing concentrations of ethanol. After blocking endogenous peroxidase with $3 \%$ hydrogen peroxidase in methanol for $10 \mathrm{~min}$ at room temperature, sections were boiled on citrate buffer for $10 \mathrm{~min}$ and incubated with $10 \%$ normal blocking serum for $20 \mathrm{~min}$. The sections were then incubated with anti-ITGA2 antibody (R\&D systems) overnight at $4{ }^{\circ} \mathrm{C}$, followed by immunodetection using the Envision System (DAKO. Glostrup, Denmark). The slides were then counterstained with hematoxylin for $1 \mathrm{~min}$ and mounted. For quantification, the expression level of ITGA2 was scored using a composite score by combining the percentage of positively stained tumor cells and the intensity of staining [10]. The percentage of positive cells was scored as: 0 , no positive tumor cells; $1,<10 \% ; 2,10-35 \% ; 3,35-75 \%$; $4,>75 \%$. The intensity of staining was scored as: 0 , no or weak staining (light yellow); 1, moderate staining (yellow brown); 2 , strong staining (brown).

CD31/PAS double immunohistochemical staining was performed to detect the presence of $\mathrm{VM}$ as we previously described [5]. For quantification, VM density (VMD) was defined as the average VM count from five $\times 400$ magnification fields per slide. The average VMD from eight tumor samples was obtained as the final VMD count.

Statistical analysis. Results were expressed as the mean \pm S.D. The random variance model $(\mathrm{RVM}) \mathrm{t}$ was used for detection of the differentially expressed genes for the 143B and HOS groups. The one-way ANOVA test was used to compare quantitative data. A value of false discovery rate (FDR) or p-value less than 0.05 was considered statistically significant.

Data availability. The microarray data are publicly available in the GEO repository under accession number GSE119975 (GEO: GSE119975). 


\section{Results}

Chronological changes of VM formation by osteosarcoma cells. A tube formation assay using Matrigel composed of ECM has been used to test the ability of tumor cells to develop a vascular phenotype, also known as VM. As shown in Figure 1, after $3 \mathrm{~h}$ the highly aggressive 143B cells on Matrigel orientated themselves towards each other and began to build up cell-to-cell links. The tubular channels developed dynamically and inosculated during an 8 to $24 \mathrm{~h}$ period. These networks consisted of clear elongated cell bodies, which formed polygonal-shape meshwork. At $24 \mathrm{~h}$, complete tubular networks have virtually established and seldomisolated cells were left out. In contrast, the lowly invasive HOS cells failed to develop tubular networks on Matrigel at any time point. In this Matrigel-based culture system, HOS cells exhibited the feature of small, round colony morphology (Figure 1). Evidence demonstrated that this assay should be finished within $24 \mathrm{~h}$ because the cells commonly undergo cell death after $24 \mathrm{~h}$, leading to tube detachment and disruption [11]. Therefore, we chose a time point of $24 \mathrm{~h}$ for subsequent study when VM formation is mature but discontinued network is not obvious.

Identification of DEGs between 143B and HOS group. Based on the cut-off criteria, a total of 907 DEGs were identified between the 143B and HOS groups, of which 381 were upregulated in 143B compared with HOS group and 526 were downregulated. Among these DEGs, MMP1 (matrix metallopeptidase 1) was the most upregulated, with an FC of 1273.01, whereas MYL9 (myosin, light chain 9, regulatory) was the most downregulated, FC being 178.57. The top 10 upregulated and downregulated DEGs are listed in Table 1. A hierarchical clustering analysis heatmap presented in Figure 2 showed a clear distinction between 143B and HOS groups.

GSEA GO analysis. To investigate underlying mechanisms involved in the VM, we used GSEA to identify gene sets and key pathways that are altered in 143B and HOS groups. The results of the GO analysis based on normalized enriched score (NES) and p-value $(<0.01)$ are shown for $143 \mathrm{~B}$ and HOS groups in Tables 2, 3 and Figure S1A, S1B. We identified GO categories including "cell adhesion", "angiogenesis", "positive regulation vascular endothelial growth factor production", "positive regulation of Wnt receptor signaling pathway", "positive regulation of endothelial cell proliferation" and "regulation of transforming growth factor beta

Table 1. The top 10 significant upregulated and downregulated DEGs in 143B versus HOS groups.

\begin{tabular}{llcl}
\hline Probe set & Gene symbol & Fold change & p-value \\
\hline Upregulated & & & \\
TC11002234.hg.1 & MMP1 & 1273.01 & $<10^{-7}$ \\
TC05000218.hg.1 & ITGA2 & 417.67 & $<10^{-7}$ \\
TC08001321.hg.1 & TRPA1 & 133.84 & $<10^{-7}$ \\
TC01003042.hg.1 & TBX15 & 121.19 & $<10^{-7}$ \\
TC15001837.hg.1 & ANPEP & 67.33 & $<10^{-7}$ \\
TC0X000954.hg.1 & SRPX & 66.83 & $<10^{-7}$ \\
TC04002931.hg.1 & PDGFRA & 46.54 & $<10^{-7}$ \\
TC09001278.hg.1 & FRMD3 & 43.01 & $<10^{-7}$ \\
TC02002098.hg.1 & LOC100996862 & 38.33 & $<10^{-7}$ \\
TC05000372.hg.1 & F2RL1 & 37.42 & $<10^{-7}$ \\
Downregulated & & & \\
TC20000268.hg.1 & MYL9 & 178.57 & $<10^{-7}$ \\
TC08001051.hg.1 & TNFRSF10D & 40.00 & $<10^{-7}$ \\
TC10000771.hg.1 & INA & 37.04 & $<10^{-7}$ \\
TC17000309.hg.1 & WSB1 & 29.41 & $<10^{-7}$ \\
TC10000415.hg.1 & SRGN & 29.41 & $<10^{-7}$ \\
TC06001271.hg.1 & ELOVL2 & 28.57 & $<10^{-7}$ \\
TC01003796.hg.1 & SLC30A1 & 27.78 & $<10^{-7}$ \\
TC0X000195.hg.1 & USP9X & 20.83 & $<10^{-7}$ \\
TC06000640.hg.1 & CENPQ & 18.87 & $<10^{-7}$ \\
TC06001782.hg.1 & MUT & 18.52 & $<10^{-7}$ \\
\hline
\end{tabular}

3 hours
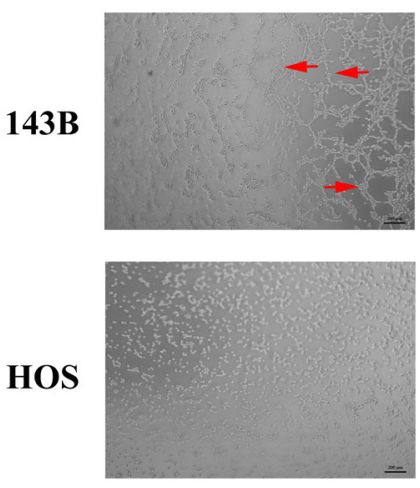

8 hours
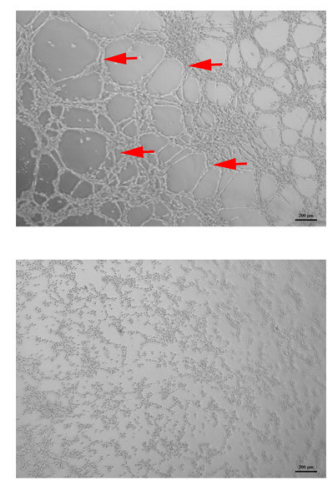

12 hours
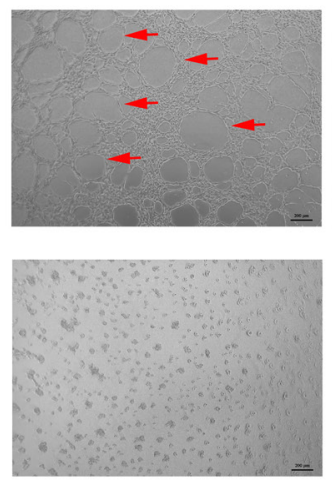

24 hours
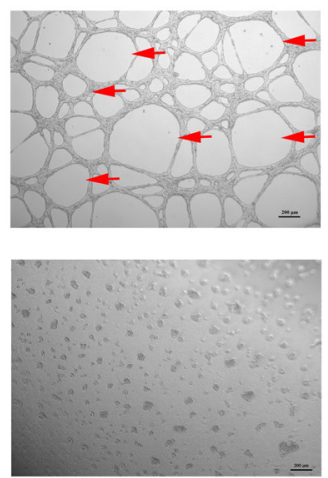

Figure 1. Kinetics of VM formation by osteosarcoma cells. Cells were plated on Matrigel and images were taken at different times indicated. 143B cells started to form VM channels as early as 3 hours after plating on Matrigel. Well-defined capillary-like tubes were observed by 24 hours. HOS cells failed to form patterned networks. Scale bar: $200 \mu \mathrm{m}$; arrows denote VMs formed by 143B cells. 
receptor signaling pathway" were enriched in 143B groups, suggesting blood vessel development and tumor progression involved in 143B groups. In HOS groups, GO categories such as "collagen fibril organization", "keratinocyte differentiation", "glycosaminoglycan biosynthetic process" were enriched, suggesting a matrix-producing phenotype in HOS cells.

GSEA PATHWAY analysis. The results of the pathway analysis based on normalized enriched score (NES) and p-value $(<0.01)$ are shown for $143 \mathrm{~B}$ and HOS groups in Tables 4, 5 and Figure S1C, S1D. We identified pathways involved in tumor development and angiogenesis such as "TGF-beta signaling pathway", "Wnt signaling pathway", "VEGF signaling pathway" and "Chemical carcinogenesis" were significantly upregulated in 143B group. In HOS group, pathways including "Type II diabetes mellitus" and "Amoebiasis" were upregulated.
Signal-net analysis. Gene signal transduction networks (Signal-net) was performed to clarify the key genes involved in VM formation in osteosarcoma. As shown in Figure 3, there was a mount of 47 key genes, which were obtained in the Signal-net. As shown in Table 6, the top 10 genes ranked according to degree value were ITGA2 (integrin subunit alpha 2), $P R K A C B$ (protein kinase cAMP-activated catalytic subunit beta), ITGA1 (integrin subunit alpha 1), ITGA6 (integrin subunit alpha 6), ACTN1 (actinin alpha 1), ACTN4 (actinin alpha 4), PLCB4 (phospholipase C beta 4), PDGFRB (platelet derived growth factor receptor beta), PDGFRA (platelet derived growth factor receptor alpha), GJA1 (gap junction protein alpha 1).

qRT-PCR validation. To validate the accuracy of the microarray, we chose a total of 14 DEGs including top five upregulated genes (MMP1, ITGA2, TRPA1, TBX15, ANPEP),

Table 2. Gene sets enriched in 143B group.

\begin{tabular}{|c|c|c|c|c|c|}
\hline Gene Set ID & Gene Set Name & Size & NES & NOM p-val & FDR q-va \\
\hline GO_0007155 & cell adhesion & 72 & 2.24 & 0 & 0.02 \\
\hline GO_0001525 & angiogenesis & 45 & 2.09 & 0.0020 & 0.06 \\
\hline GO_0010575 & $\begin{array}{l}\text { positive regulation vascular endothelial growth factor } \\
\text { production }\end{array}$ & 6 & 2.07 & 0 & 0.06 \\
\hline GO_0030177 & positive regulation of Wnt receptor signaling pathway & 6 & 1.91 & 0.0020 & 0.14 \\
\hline GO_0001938 & positive regulation of endothelial cell proliferation & 9 & 1.89 & 0.0059 & 0.15 \\
\hline GO_0017015 & $\begin{array}{l}\text { regulation of transforming growth factor beta receptor } \\
\text { signaling pathway }\end{array}$ & 6 & 1.84 & 0.0043 & 0.21 \\
\hline
\end{tabular}

NES, normalized enrichment score; NOM p-value, nominal p value; FDR q-val, false discovery rate q value.

Table 3. Gene sets enriched in HOS group.

\begin{tabular}{|c|c|c|c|c|c|}
\hline Gene Set ID & Gene Set Name & Size & NES & NOM p-val & FDR q-val \\
\hline GO_0030199 & collagen fibril organization & 12 & -2.59 & 0 & 0.00 \\
\hline GO_0030216 & keratinocyte differentiation & 10 & -1.96 & 0 & 0.35 \\
\hline GO_0043434 & response to peptide hormone stimulus & 10 & -1.96 & 0.0040 & 0.24 \\
\hline GO_0006024 & glycosaminoglycan biosynthetic process & 6 & -1.95 & 0 & 0.20 \\
\hline GO_0070509 & calcium ion import & 4 & -1.89 & 0 & 0.29 \\
\hline
\end{tabular}

NES, normalized enrichment score; NOM p-value, nominal p value; FDR q-val, false discovery rate q value.

Table 4. Signaling pathways enriched in $143 B$ group.

\begin{tabular}{|c|c|c|c|c|c|}
\hline Gene Set ID & Gene Set Name & Size & NES & NOM p-val & FDR q-val \\
\hline PATH4350 & TGF-beta signaling pathway & 16 & 2.19 & 0 & 0.02 \\
\hline PATH4310 & Wnt signaling pathway & 21 & 2.08 & 0.0020 & 0.03 \\
\hline PATH4370 & VEGF signaling pathway & 8 & 1.96 & 0.0020 & 0.06 \\
\hline PATH5204 & Chemical carcinogenesis & 3 & 1.67 & 0.0082 & 0.22 \\
\hline
\end{tabular}

NES, normalized enrichment score; NOM p-value, nominal p value; FDR q-val, false discovery rate q value.

Table 5. Signaling pathways enriched in HOS group.

\begin{tabular}{llcccc}
\hline Gene Set ID & Gene Set Name & Size & NES & NOM p-val & FDR q-val \\
\hline PATH5146 & Amoebiasis & 20 & -1.84 & 0.0060 & 0.70 \\
PATH4930 & Type II diabetes mellitus & 3 & -1.71 & 0.0021 & 0.62 \\
\hline
\end{tabular}

NES, normalized enrichment score; NOM p-value, nominal p value; FDR q-val, false discovery rate q value. 
A

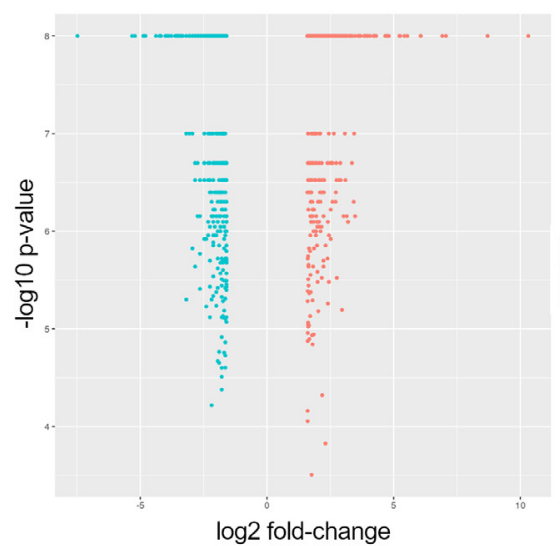

B

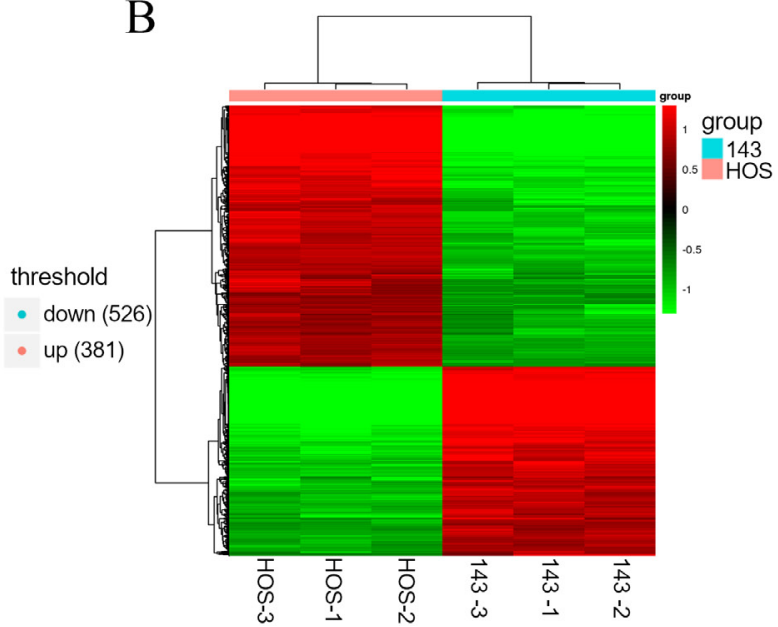

Figure 2. Microarray gene expression profiling of 143B vs. HOS groups. A) Volcano plot shows differentially expressed genes comparing 143B and HOS groups. Red dots represent genes overexpressed in 143B group (fold change $\geq 3$, p-value $<0.05$ ). Blue dots represent genes underexpressed in 143B group (fold change $\geq 3$, p-value $<0.05$ ). B) Hierarchical clustering analysis heat-map shows differentially expressed genes in $143 \mathrm{~B}$ and HOS groups. Red represents upregulated genes and green represents downregulated genes.

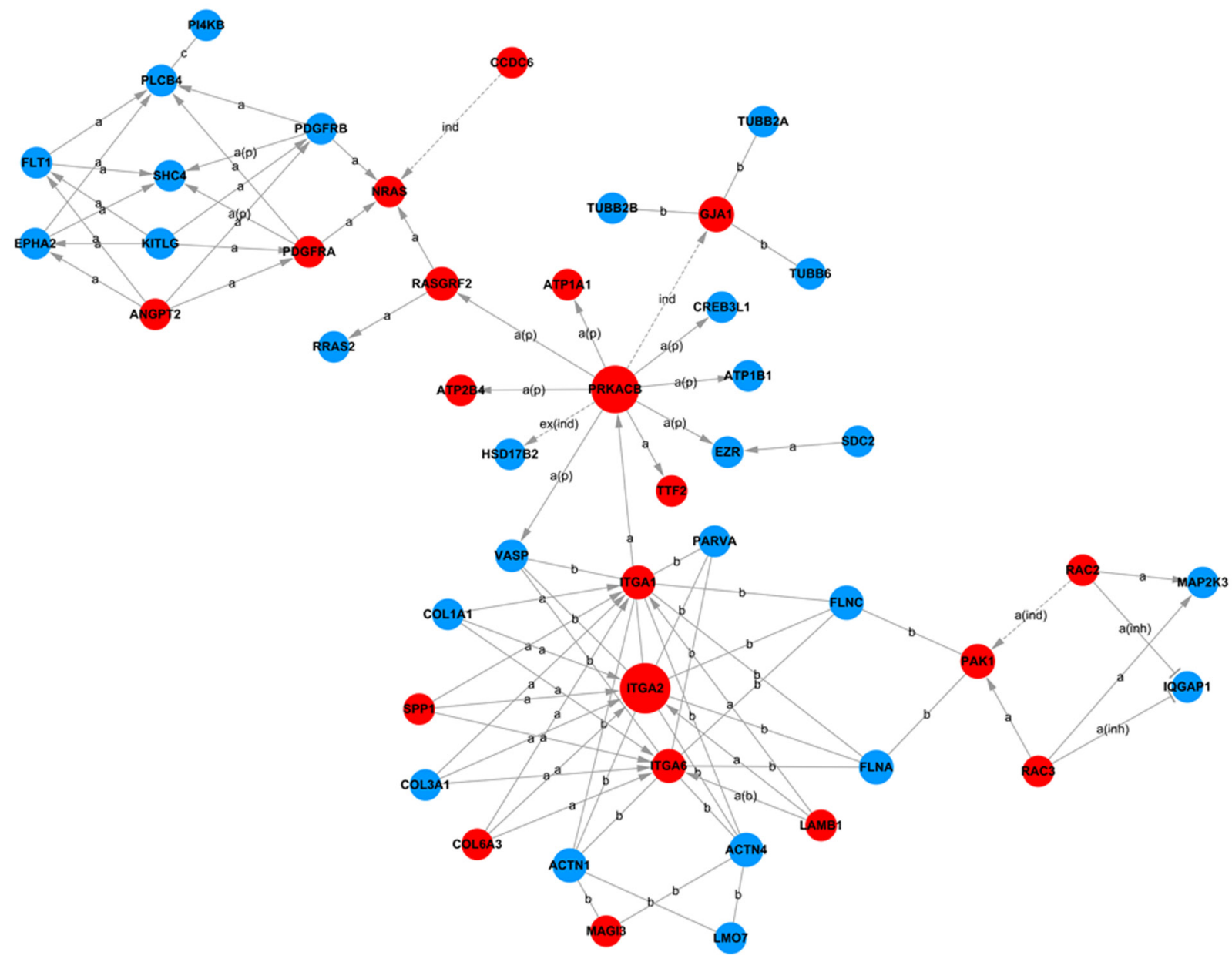

Figure 3. Signal-net analysis of DEGs. The red circle represents the upregulated DEGs and the blue circles downregulated DEGs. The area of the circle represents the betweenness centrality. Interaction between the genes is shown as a: activation, a(inh): activation (inhibition), a(b): activation(binding/ association), a(p): activation(phosphorylation), b: binding/association, c: compound, ex(ind): expression (indirect effect), ind: indirect effect. 


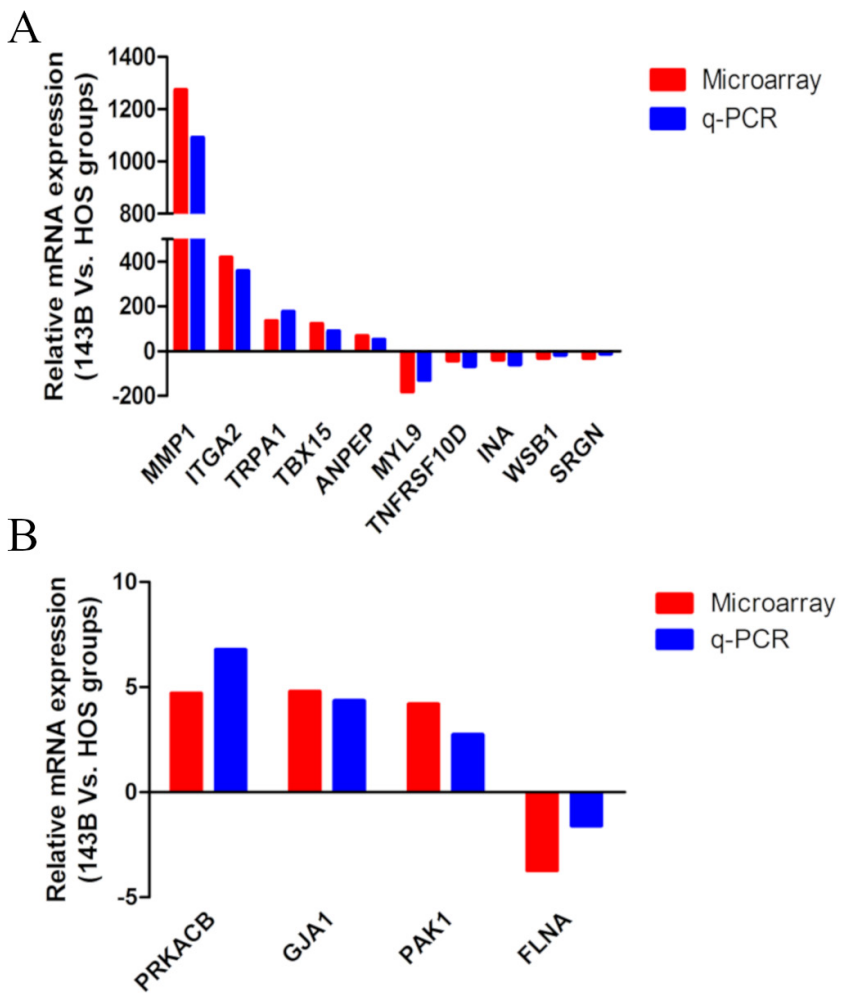

Figure 4. qRT-PCR of dysregulated mRNA shows the same regulation of mRNA with microarray results. A) Ten dysregulated mRNAs were chosen for being the top upregulated and downregulated mRNAs. B) Four dysregulated $\mathrm{mRNAs}$ were chosen for being the top four key genes identified in Signal-net analysis. ${ }^{\star} \mathrm{p}<0.05$ for each mRNA.

Table 6. Top 10 DEGs identified by Signal-net analysis.

\begin{tabular}{llccc}
\hline $\begin{array}{l}\text { Gene } \\
\text { symbol }\end{array}$ & Description & Degree & Indegree & Outdegree \\
\hline ITGA2 & integrin subunit alpha 2 & 12 & 11 & 7 \\
PRKACB & $\begin{array}{l}\text { protein kinase cAMP-acti- } \\
\text { vated catalytic subunit beta }\end{array}$ & 11 & 1 & 10 \\
ITGA1 & integrin subunit alpha 1 & 11 & 11 & 6 \\
ITGA6 & integrin subunit alpha 6 & 11 & 11 & 6 \\
ACTN1 & actinin alpha 1 & 5 & 5 & 5 \\
ACTN4 & actinin alpha 4 & 5 & 5 & 5 \\
PLCB4 & phospholipase C beta 4 & 5 & 5 & 1 \\
PDGFRB & $\begin{array}{l}\text { platelet derived growth } \\
\text { factor receptor beta } \\
\text { platelet derived growth }\end{array}$ & 5 & 2 & 3 \\
factor receptor alpha & 5 & 2 & 3 \\
GJA1 & $\begin{array}{l}\text { gap junction protein } \\
\text { alpha 1 }\end{array}$ & 4 & 4 & 3 \\
\hline
\end{tabular}

top five downregulated genes (MYL9, TNFRSF10D, INA, WSB1, SRGN) and four top key genes (PRKACB, ITGA1, ITGA6, ACTN1) identified in Signal-net analysis for qRT-PCR verification. Expression levels of these 14 DEGs were approximately consistent with the microarray results (Figure 4).
ITGA2 knockdown inhibited VM formation by 143B cells in vitro and in vivo. Hub genes are defined as a small ratio of nodes with the highest connectivity with other genes in the constructed gene network and often possess biological activity as well. We chose the top one hub gene ITGA2, which was also ranked second highest among upregulated DEGs to evaluate its role in osteosarcoma VM formation.

Firstly, we used siRNA to silence ITGA2 to test its impact on in vitro VM in $143 \mathrm{~B}$ cell lines. As shown in Figure 5A and $5 \mathrm{~B}$, two siRNAs significantly decreased the levels of ITGA2 mRNA and protein in comparison with control cells transfected with negative control siRNA. Secondly, in vitro $\mathrm{VM}$ assay showed that a knockdown of ITGA2 resulted in a significant decrease of VM channels in 143B cells (Figure 5C). To evaluate the effect of ITGA2 knockdown in vivo, ITGA2 KD 143B cells were intratibially inoculated in the left tibia of the nude mice. ITGA2 KD group formed smaller tumors at 30 days post-implantation when compared to NC group (Figure 5D). Primary tumor growth was inhibited in ITGA2 KD group compared to NC group during 30 days (Figure 5E). qPCR and immunohistochemical assays were performed to confirm the efficacy of ITGA2 knockdown in tumor tissues. As anticipated, ITGA2 KD group displayed reduced ITGA2 expression (Figures 5F, 5H, 5I). Thirdly, ITGA2 knockdown resulted in significant decreases in the number of lung metastatic nodules (Figures 5D, 5G). H\&E staining of the primary tumors and lungs tissues showed more osteosarcoma cells with strongly stained enlarged nucleoli in ITGA2 $\mathrm{KD}$ group than in NC group (Figure $5 \mathrm{H}$ ). Tumors in ITGA2 KD group showed reduced VMD compared with NC group (Figure 5J). Collectively, these data demonstrate that ITGA2 knockdown can suppress osteosarcoma VM and metastasis.

\section{Discussion}

Osteosarcoma is the most frequent primary malignant bone tumor mainly affecting adolescents and children with a high propensity for fatal metastases. The 5 -year survival rate of patients with localized osteosarcoma has increased from $20 \%$ to $70 \%$ due to the introduction of neo-adjuvant chemotherapy combined with increasingly improved surgical approaches [12]. However, patients with metastases, still have poor prognoses, with overall survival rates of approximately $20 \%$ [13]. Consequently, it is crucial to identify the mechanism of metastasis in osteosarcoma for more effective treatment. Due to VM's contribution to distant metastasis and poor clinical outcome, which we have previously reported [4], it is of great importance to dissect the molecules involved in mediating osteosarcoma VM.

To our knowledge, this study is the first attempt to provide a genome-wide transcriptional comparative analysis between VM-positive and VM-negative osteosarcoma cells. We obtained many DEGs between 143B and HOS groups, among which, several showed remarkable fold change such as MMP1, ITGA2 and MYL9. Reports show that these DEGs 


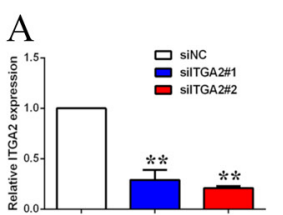

B

D

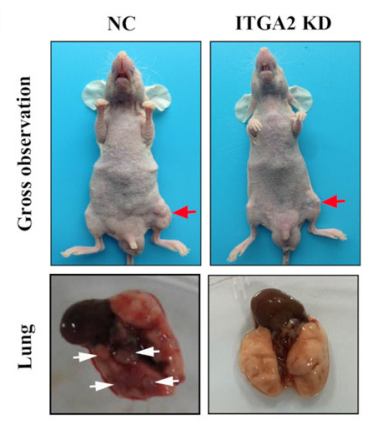

$\mathrm{H}$
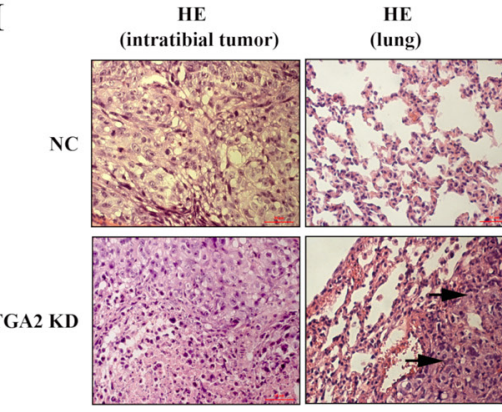

$\mathrm{E}$

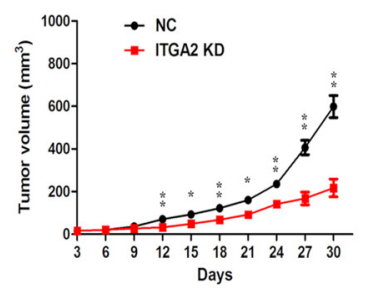

$\mathrm{F}$

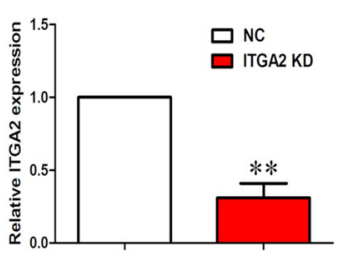

C
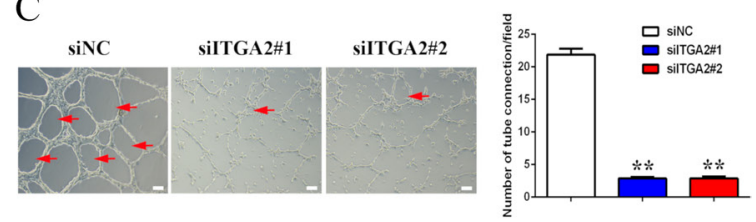

G

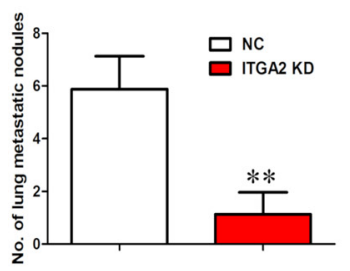

$\mathrm{J}$

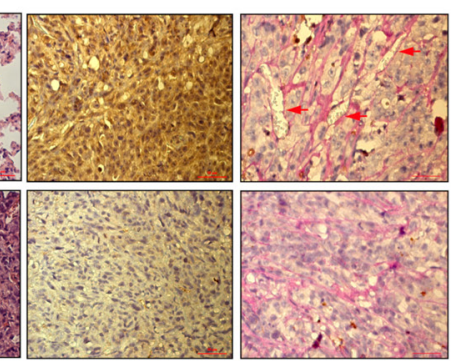

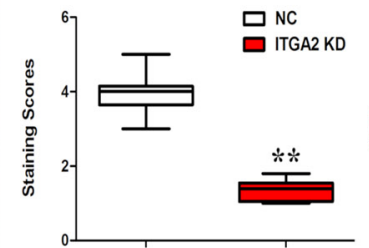

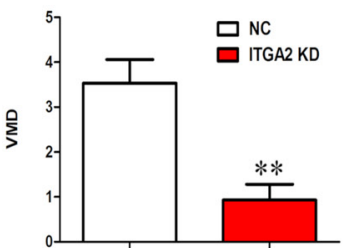

Figure 5. ITGA2 knockdown inhibited osteosarcoma VM formation and metastasis. The mRNA and protein expression of ITGA2 in ITGA2-knockdown 143B cells were detected using $\mathrm{qRT}$-PCR (A) and western blot (B). C): In vitro VM formation in ITGA2-knockdown 143B cells was analyzed using Matrigel-based tube formation assay. Red arrows denote VM channels formed by 143B cells. The results are expressed as the mean \pm SD of triplicate samples. Scale bars $=100 \mu \mathrm{m}$. D): The ITGA2-knockdown 143B cells were injected intratibially into BALB/C nude mice. After 30 days, the mice and lungs were photographed. Red arrows denote 143B-injected limbs and white arrows denote metastatic nodules on the lung surface. E): Intratibial tumor volumes measured with calipers at the indicated time points. ${ }^{*} p<0.05,{ }^{* *} p<0.01$. F): ITGA2 mRNA expression was detected in intratibial tumors from ITGA2 KD group or NC group using $\mathrm{QRT}$-PCR. G): The number of lung metastatic nodules in ITGA2 KD group and NC group. $\mathrm{H})$ : Representative images of $\mathrm{H} \& \mathrm{E}$ and immunohistochemical staining of the resected tumor and lung from ITGA2 KD group and NC group on day 30 post-inoculation. Black arrows denote micrometastatic tumors. Red arrows denote VM channels. I) Quantitative analysis of ITGA2 staining. J): Quantification of VMD in ITGA2 KD group and NC group. Scale bars $=50 \mu \mathrm{m}$. The results are expressed as the mean $\pm \mathrm{SD}$ of eight samples. ${ }^{* *} \mathrm{p}<0.01$ compared to the NC group.

may contribute to VM formation. MMP1 lists on the top of upregulated DEGs, consistent with previous findings demonstrating enhanced expression of MMP1 in 143B cell which plays a central role in the development of pulmonary metastases in osteosarcomas [14]. MMP14 have been implicated in VM formation in various carcinomas, making it potential target for anti-VM therapy [15]. Our result also showed that MMP14 is upregulated by 3.18-fold in 143B group as compared to HOS group. But more imposingly, $M M P 1$ was upregulated much more dramatically than $M M P 14$ did in 143B group, implying the potential importance of $M M P 1$ in addition to MMP14 for VM formation in osteosarcomas. ITGA2 encodes a heterodimeric transmembrane receptor that mediates cell-ECM binding. Since interactions between tumor cells and their ECM-rich surroundings are necessary for VM, reports demonstrated that integrins like ITGB1,
ITGAV have been proven to participate in VM formation [16]. Increased ITGA2 expression is also associated with increased osteosarcoma metastasis and invasion [17], suggesting its involvement in VM formation in osteosarcoma. MYL9 lists on the top of downregulated DEGs. The decreased expression of MYL9 represents stromal loss in tumor microenvironments [18], which could play a role in VM formation [2]. In this context, the impressive downregulation of MYL9 in our finding may lead to an increased VM phenotype. Taken together, we hypothesized that these DEGs have the potential to be biomarkers for osteosarcoma VM.

GO categories like "collagen fibril organization", "calcium ion import" and "glycosaminoglycan biosynthetic process" was significantly upregulated in HOS compared to 143B groups, which is consistent with the fact that osteoblast-like HOS cells have an activity of bone formation [19]. Because 
VM refer to the blood tubules formed by highly aggressive tumor cells, our finding of this non-metastatic, well-differentiated phenotype of HOS cells may explain why HOS were incapable of VM.

The increased expression level of "angiogenesis" and "positive regulation VEGF production" GO categories are in accordance with the enriched "VEGF signaling pathway" in 143B compared to HOS groups. Some pro-angiogenic molecules are crucial in tumor angiogenesis and equally have important function in VM formation [20]. In our results, PTGS2 in the above-mentioned GO and pathway categories has fold change of 6.65. It is well known that PTGS2 encodes a key enzyme COX-2 that generates $\mathrm{PGE}_{2}$ that binds to prostanoid receptor and promotes tumor VM [21]. Additionally, COX-2 overexpression in osteosarcoma patients correlates with the occurrence of lung metastasis [22]. Based on the results of our study and others, we suggest that PTGS2 might contribute to the development of VM in osteosarcoma.

"TGF-beta signaling pathway" was significantly upregulated in 143B compared to HOS groups, which is consistent with the GO category "regulation of TGF- $\beta$ receptor signaling pathway" enriched in 143B. Bone morphogenic proteins (BMPs) constitute the largest member in TGF- $\beta$ superfamily. BMPs are overexpressed in osteosarcoma and correlated with poor prognosis in bone tumors [23]. Study showed an important role of BMPs in supporting VM in metastatic tumor cell line [24]. Here, we demonstrated 4.74-fold change of BMP6 in 143B compared with HOS, implying the possible involvement of BMP6 in osteosarcoma VM.

GSEA pathway "Wnt signaling pathway" and GO "positive regulation of Wnt receptor signaling pathway" were upregulated in 143B group. Wnt signaling has been demonstrated to play an important role in VM in several studies [25]. Aberrantly activated Wnt pathway plays a key role in the development and metastasis of osteosarcoma and drugs targeting to inactivate this pathway are useful therapeutic application to inhibit osteosarcoma [26]. Thus, we can speculate that the significantly upregulated Wnt signaling pathway may facilitate osteosarcoma VM.

Signal-net analysis was performed to identify hub genes. ITGA2, listed as one of the top ten upregulated DEGs, also has the highest degree identified by signal-net analysis. The hub genes, which have many neighbors, are much more likely to be essential genes compared to non-hub genes. Thus, we herein focus our attention on the role of ITGA2 in osteosarcoma VM. The loss-of-functional study demonstrated that knockdown of ITGA2 lead to VM destruction both in vitro and in vivo, demonstrating the promoting role of ITGA2 in VM formation. More importantly, VM functions as an alternative microcirculation to transmit blood fluid in tumor tissues and provide an escape route for new tumors in distant organs. Indeed, knockdown of ITGA2 inhibited primary tumor growth as well as lung metastatic nodules in metastatic orthotropic-transplant mice model. These findings were consistent with previous studies that the expression of ITGA2 was associated with metastatic behaviors in osteosarcoma cells and other malignancies [27, 28]. ITGA2 were involved in GO category "cell adhesion". As mentioned before, integrins function as cell adhesion receptors for ECM proteins. Integrin-mediated cell adhesion activates a cytoplasmic protein tyrosine kinase called FAK, which has been recognized as a key factor in VM [28]. In our earlier findings, FAK was found to be activated in osteosarcoma VM [4]. We can speculate that ITGA2 may promote VM formation of osteosarcoma by activating FAK signaling. Further studies are needed to explore this issue.

In conclusion, as far as we know, we showed for the first time the differential mRNAs expression profiles associated with VM phenotype in osteosarcoma cells. The enrichedGSEA GO terms and PATHWAY in 143B group indicated the possibility of their involvement in osteosarcoma VM formation. Furthermore, signal-net analysis identified key gene ITGA2 with the highest degrees. Functionally, knockdown of ITGA2 inhibited osteosarcoma VM formation as well as osteosarcoma growth and metastasis, suggesting its potential role in OS VM and metastasis. Given the crucial role of $\mathrm{VM}$ in poor prognosis for osteosarcoma, our datasets are expected to help elucidate the molecules engaged in osteosarcoma VM and determine potential targets for inhibiting metastasis of osteosarcoma.

Supplementary information is available in the online version of the paper.

Acknowledgments: This study was supported by the National Natural Science Foundation of China (No. 81903851, 81673017, 81572188, 21502014), the Jiangsu Province Natural Science Foundation (No. BK20191084, SBK2019040340, BK2012775), the Fundamental Research Funds for the Central Universities (No.2242016k40152), the Liaoning Province Natural Science Foundation (201602114) and the Research Foundation of Educational Committee of Liaoning Province (JDL2017027).

\section{References}

[1] MANIOTIS AJ, FOLBERG R, HESS A, SEFTOR EA, GARDNER LM et al. Vascular channel formation by human melanoma cells in vivo and in vitro: vasculogenic mimicry. Am J Pathol 1999; 155: 739-752. https://doi.org/10.1016/ S0002-9440(10)65173-5

[2] HENDRIX MJ, SEFTOR EA, HESS AR, SEFTOR RE. Vasculogenic mimicry and tumour-cell plasticity: lessons from melanoma. Nat Rev Cancer 2003; 3: 411-421. https://doi. org/10.1038/nrc1092

[3] FOLBERG R, ARBIEVA Z, MOSES J, HAYEE A, SANDAL $\mathrm{T}$ et al. Tumor cell plasticity in uveal melanoma: microenvironment directed dampening of the invasive and metastatic genotype and phenotype accompanies the generation of vasculogenic mimicry patterns. Am J Pathol 2006; 169: 13761389. https://doi.org/10.2353/ajpath.2006.060223 
[4] REN K, YAO N, WANG G, TIAN L, MA J et al. Vasculogenic mimicry: a new prognostic sign of human osteosarcoma. Hum Pathol 2014; 45: 2120-2129. https://doi.org/10.1016/j. humpath.2014.06.013

[5] YAO N, REN K, WANG Y, JIN Q, LU X et al. Paris polyphylla Suppresses Proliferation and Vasculogenic Mimicry of Human Osteosarcoma Cells and Inhibits Tumor Growth In Vivo. Am J Chin Med 2017; 45: 575-598. https://doi. org/10.1142/s0192415x17500343

[6] REN K, ZHANG J, GU X, WU S, SHI X et al. Migrationinducing gene-7 independently predicts poor prognosis of human osteosarcoma and is associated with vasculogenic mimicry. Exp Cell Res 2018; 369: 80-89. https://doi. org/10.1016/j.yexcr.2018.05.008

[7] KIRSCHMANN DA, SEFTOR EA, HARDY KM, SEFTOR RE, HENDRIX MJ. Molecular pathways: vasculogenic mimicry in tumor cells: diagnostic and therapeutic implications. Clin Cancer Res 2012; 18: 2726-2732. https://doi. org/10.1158/1078-0432.ccr-11-3237

[8] PAULIS YW, SOETEKOUW PM, VERHEUL HM, TJANHEIJNEN VC, GRIFFIOEN AW. Signalling pathways in vasculogenic mimicry. Biochim Biophys Acta 2010; 1806: 18-28. https://doi.org/10.1016/j.bbcan.2010.01.001

[9] HENDRIX MJ, SEFTOR EA, SEFTOR RE, CHAO JT, CHIEN DS et al. Tumor cell vascular mimicry: Novel targeting opportunity in melanoma. Pharmacol Ther 2016; 159: 83-92. https://doi.org/10.1016/j.pharmthera.2016.01.006

[10] BEHNSAWY HM, MIYAKE H, ABDALLA MA, SAYED MA, AHMED EFI et al. Expression of integrin proteins in nonmuscle-invasive bladder cancer: significance of intravesical recurrence after transurethral resection. BJU Int 2011; 107: 240-246 https://doi.org/10.1111/j.1464-410X.2010.09534.x

[11] ARNAOUTOVA I, KLEINMAN HK. In vitro angiogenesis: endothelial cell tube formation on gelled basement membrane extract. Nat Protoc 2010; 5: 628-635. https://doi. org/10.1038/nprot.2010.6

[12] ANDO K, HEYMANN MF, STRESING V, MORI K, REDINI F et al. Current therapeutic strategies and novel approaches in osteosarcoma. Cancers (Basel) 2013; 5: 591-616. https:// doi.org/10.3390/cancers5020591

[13] MILLER BJ, CRAM P, LYNCH CF, BUCKWALTER JA. Risk factors for metastatic disease at presentation with osteosarcoma: an analysis of the SEER database. J Bone Joint Surg Am 2013; 95: e89. https://doi.org/10.2106/jbjs.1.01189

[14] KIMURA R, ISHIKAWA C, ROKKAKU T, JANKNECHT R, MORI N. Phosphorylated c-Jun and Fra-1 induce matrix metalloproteinase- 1 and thereby regulate invasion activity of 143B osteosarcoma cells. Biochim Biophys Acta 2011; 1813: 1543-1553. https://doi.org/10.1016/j.bbamcr.2011.04.008

[15] SEFTOR RE, SEFTOR EA, KOSHIKAWA N, MELTZER PS, GARDNER LM et al. Cooperative interactions of laminin 5 gamma2 chain, matrix metalloproteinase-2, and membrane type-1-matrix/metalloproteinase are required for mimicry of embryonic vasculogenesis by aggressive melanoma. Cancer Res 2001; 61: 6322-6327.
[16] RUFFINI F, GRAZIANI G, LEVATI L, TENTORI L, D'ATRI $S$ et al. Cilengitide downmodulates invasiveness and vasculogenic mimicry of neuropilin 1 expressing melanoma cells through the inhibition of alphavbeta5 integrin. Int J Cancer 2015; 136: E545-558. https://doi.org/10.1002/ijc.29252

[17] LIU X, LIANG Z, GAO K, LI H, ZHAO G et al. MicroRNA-128 inhibits EMT of human osteosarcoma cells by directly targeting integrin alpha2. Tumour Biol 2016; 37: 79517957. https://doi.org/10.1007/s13277-015-4696-0

[18] HUANG YQ, HAN ZD, LIANG YX, LIN ZY, LING XH et al. Decreased expression of myosin light chain MYL9 in stroma predicts malignant progression and poor biochemical recurrence-free survival in prostate cancer. Med Oncol 2014; 31 : 820. https://doi.org/10.1007/s12032-013-0820-4

[19] TAKITOH T, KATO Y, NAKASU A, TADOKORO M, BESS$\mathrm{HO} \mathrm{M}$ et al. In vitro osteogenic differentiation of HOS cells on two types of collagen gels. J Biosci Bioeng 2010; 110: 471478. https://doi.org/10.1016/j.jbiosc.2010.04.009

[20] SMITH SJ, WARD JH, TAN C, GRUNDY RG, RAHMAN R. Endothelial-like malignant glioma cells in dynamic three dimensional culture identifies a role for VEGF and FGFR in a tumor-derived angiogenic response. Oncotarget 2015; 6: 22191-22205. https://doi.org/10.18632/oncotarget.4339

[21] RONG X, HUANG B, QIU S, LI X, HE L et al. Tumor-associated macrophages induce vasculogenic mimicry of glioblastoma multiforme through cyclooxygenase-2 activation. Oncotarget 2016; 7: 83976-83986. https://doi.org/10.18632/ oncotarget.6930

[22] QU L, LIU B. Cyclooxygeanse-2 promotes metastasis in osteosarcoma. Cancer Cell Int 2015; 15: 69. https://doi. org/10.1186/s12935-015-0220-2

[23] MUNDY C, YANG E, TAKANO H, BILLINGS P, PACIFICI M. Heparan sulfate antagonism alters bone morphogenetic protein signaling and receptor dynamics, suggesting a mechanism in hereditary multiple exostoses. J Biol Chem 2018; 293: 7703-7716. https://doi.org/10.1074/jbc.RA117.000264

[24] AZAD T, GHAHREMANI M, YANG X. The Role of YAP and TAZ in Angiogenesis and Vascular Mimicry. Cells 2019; 8: E407. https://doi.org/10.3390/cells8050407

[25] QI L, SONG W, LIU Z, ZHAO X, CAO W et al. Wnt3a Promotes the Vasculogenic Mimicry Formation of Colon Cancer via Wnt/beta-Catenin Signaling. Int J Mol Sci 2015; 16: 18564-18579. https://doi.org/10.3390/ijms160818564

[26] YANG X, WANG L, WANG Q, LI L, FU Y et al. MiR-183 inhibits osteosarcoma cell growth and invasion by regulating LRP6-Wnt/beta-catenin signaling pathway. Biochem Biophys Res Commun 2018; 496: 1197-1203. https://doi. org/10.1016/j.bbrc.2018.01.170

[27] CHUANG YC, WU HY, LIN YL, TZOU SC, CHUANG CH et al. Blockade of ITGA2 Induces Apoptosis and Inhibits Cell Migration in Gastric Cancer. Biol Proced Online 2018; 20: 10. https://doi.org/10.1186/s12575-018-0073-x

[28] HESS AR, POSTOVIT LM, MARGARYAN NV, SEFTOR EA, SCHNEIDER GB et al. Focal adhesion kinase promotes the aggressive melanoma phenotype. Cancer Res 2005; 65: 9851-9860. https://doi.org/10.1158/0008-5472.can-05-2172 


\title{
Identification of potential crucial genes associated with vasculogenic mimicry in human osteosarcoma based on gene expression profile
}

\author{
N. YAO ${ }^{1,2, *}$, K. REN ${ }^{3, *}$, X. J. GU ${ }^{4}$, S. J. WU ${ }^{5}$, X. SHI ${ }^{5}$, Q. CHANG ${ }^{3}$, Y.G. LI ${ }^{3}$, Z. X. GAO ${ }^{3}$, Q. M. JIN ${ }^{1,2}$, J. ZHANG ${ }^{1,2}$, C. WANG ${ }^{3, *}$, J. ZHOU ${ }^{1,2,6 *}$
}

\section{Supplementary Information}

$\mathrm{A}_{\mathrm{a}}$

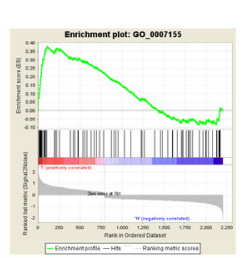

d

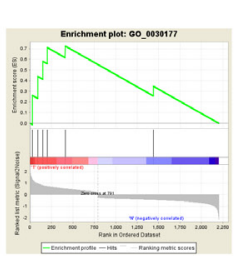

$\mathrm{B}$ a

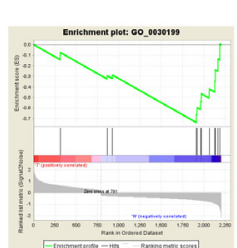

d

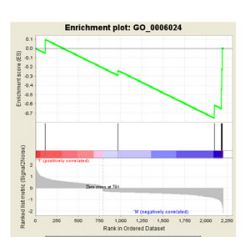

$\mathrm{C}_{\mathrm{a}}$

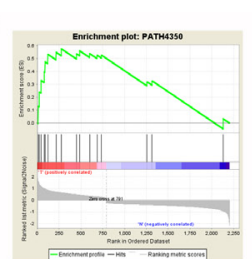

d

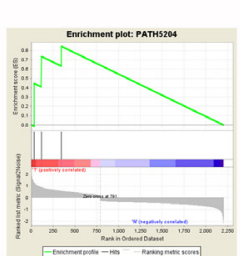

$\mathrm{D}_{\mathrm{a}}$

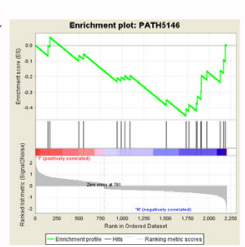

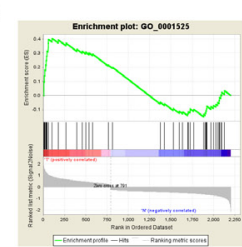

e
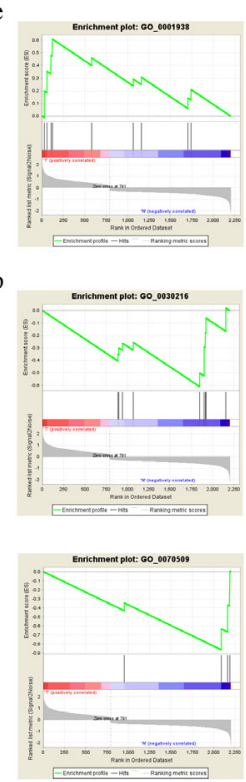

b
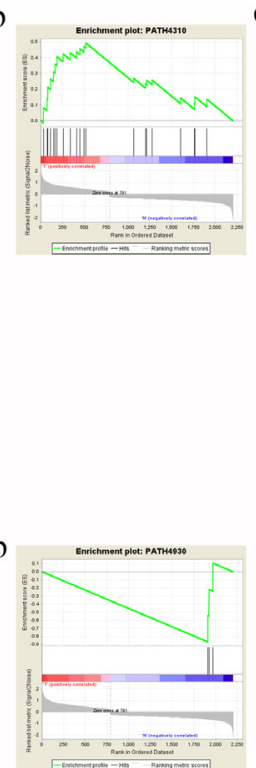
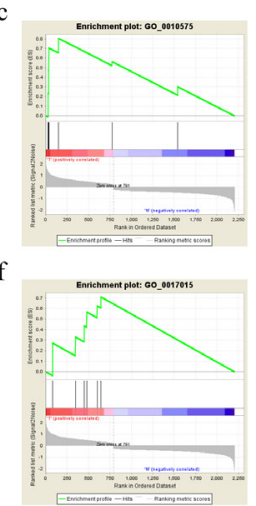

c
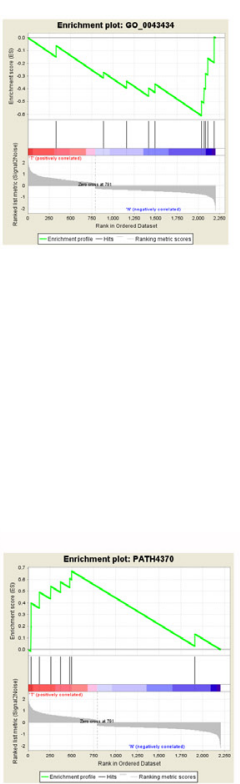

Supplementary Table S1. Primers used in qRT-PCR analysis.

\begin{tabular}{|c|c|c|}
\hline Gene & & Sequence $\left(5^{6}->3^{c}\right)$ \\
\hline \multirow[t]{2}{*}{$M M P 1$} & Forward & GGCAAATCTGGCGTGTAA \\
\hline & Reverse & GGCTGAAAGTGACTGGGAAAC \\
\hline \multirow[t]{2}{*}{ ITGA2 } & Forward & TACCCAAGAACTGCTATGC \\
\hline & Reverse & CGACGAAGTGCTACGAAA \\
\hline \multirow[t]{2}{*}{ TRPA1 } & Forward & CTACCAGAATAGGACGATA \\
\hline & Reverse & ATAGACCCAGTGGAGAAG \\
\hline \multirow[t]{2}{*}{ TBX15 } & Forward & TCCAGTTCTGTTTCTCCC \\
\hline & Reverse & ACCCACTAAGCCTGTTCC \\
\hline \multirow[t]{2}{*}{ ANPEP } & Forward & TCTTCGTCGTAGTTCACCC \\
\hline & Reverse & GCCCATCACATCCATCAG \\
\hline \multirow[t]{2}{*}{ MYL9 } & Forward & CATCTCGTCCACTTCCTCATC \\
\hline & Reverse & AACGCCTTTGCCTGCTTC \\
\hline \multirow[t]{2}{*}{ TNFRSF10D } & Forward & ACGATGAAGACGACGAAC \\
\hline & Reverse & GGGACGATTTCTGATTGA \\
\hline \multirow[t]{2}{*}{ INA } & Forward & TCAGGTTGGCAAACTTGGACT \\
\hline & Reverse & AGGTGGACGTGACTGTGGCT \\
\hline \multirow[t]{2}{*}{ WSB1 } & Forward & AACCCTGTAGCAAGAAGT \\
\hline & Reverse & ATAAGCCTCGTGAACATA \\
\hline \multirow[t]{2}{*}{ SRGN } & Forward & CCTGTTCCATTTCCGTTA \\
\hline & Reverse & TGAATCGTATCTTCCCACTT \\
\hline \multirow[t]{2}{*}{ PRKACB } & Forward & CTGAAGTGGGATGGGAAT \\
\hline & Reverse & CAAGGGCTACAATAAGGC \\
\hline \multirow[t]{2}{*}{ GJA1 } & Forward & CCAGGAGGAGACATAGGC \\
\hline & Reverse & TTCAAGGGCGTTAAGGAT \\
\hline \multirow[t]{2}{*}{ PAK1 } & Forward & GCATTCCCGTAAACTCCC \\
\hline & Reverse & TTCTAAACСТCTGCCTCCAA \\
\hline \multirow[t]{2}{*}{ FLNA } & Forward & CAATCAGGTAAACGCCATTC \\
\hline & Reverse & TCAGGAGTCAGGGCTAAAGG \\
\hline \multirow[t]{2}{*}{ GAPDH } & Forward & GGACCTGACCTGCCGTCTAG \\
\hline & Reverse & GTAGCCCAGGATGCCCTTGA \\
\hline
\end{tabular}

Supplementary Figure S1. GSEA enrichment plots in 143B group and HOS group. A) GSEA GO analysis in 143B group: (a) cell adhesion (b) angiogenesis (c) positive regulation vascular endothelial growth factor production (d) positive regulation of Wnt receptor signaling pathway (e) positive regulation of endothelial cell proliferation (f) regulation of transforming growth factor beta receptor signaling pathway. B) GSEA GO analysis in HOS group: (a) collagen fibril organization (b) keratinocyte differentiation (c) response to peptide hormone stimulus (d) glycosaminoglycan biosynthetic process (e) calcium ion import. C) GSEA PATHWAY analysis in 143B group: (a) TGF-beta signaling pathway (b) Wnt signaling pathway (c) VEGF signaling pathway (d) Chemical carcinogenesis. D) GSEA PATHWAY analysis in HOS group: (a) Amoebiasis (b) Type II diabetes mellitus. 